\title{
On the Dichotomy of a System of Linear Differential Equations with Conditionally Periodic Coefficients
}

\author{
Yu. D. Kozlov \\ Ural State University, Yekaterinburg, Russia \\ Received April 23, 2012
}

\begin{abstract}
We show that a system of linear differential equations with conditionally periodic coefficients is exponentially dichotomous if and only if the spectrum of the monodromy operator does not meet the unit circle.
\end{abstract}

DOI: $10.1134 /$ S0012266113030026

\section{INTRODUCTION}

Consider a system of linear differential equations with conditionally periodic coefficients,

$$
x^{\prime}=a(t) x,
$$

where $x$ is an $n$-vector, and $a(t)$ is a continuous conditionally periodic $n \times n$ matrix. The latter means [1] that there exists a continuous $\omega$-periodic matrix $A(\varphi), \varphi=\left(\varphi_{1}, \ldots, \varphi_{m}\right)$, $\omega=\left(\omega_{1}, \ldots, \omega_{m}\right), m \geq 2$, such that the frequencies $\beta_{i}=2 \pi / \omega_{i}$ are rationally incommensurable and $a(t)=A(e t)$ with the $m$-vector $e=(1, \ldots, 1)$.

In the present paper, we analyze the conditionally periodic system (0.1) using an approach in which this system is replaced by a system of partial differential equations [2] (or a system of integral equations [3]). The diagonal function $x(t)=u($ et $)$ of a solution $u=u(\varphi)$ of the new system is a solution of system (0.1). This permits one to define the monodromy operator [3] of system (0.1), which is an analog of the monodromy matrix of a periodic system.

Dichotomy problems for almost periodic and periodic systems of differential equations were considered in the monographs $[4,5]$. The main result of the present paper, Theorem 2.1, is an analog of the classical dichotomy theorem for a periodic system [5, p. 288].

The monodromy operator belongs to the class of weighted shift operators considered in the monograph [6]. The results of this monograph imply that the spectrum of the monodromy operator is invariant under rotations around zero (Lemma 1.1 of the present paper).

Consider the matrix equation

$$
X(\varphi)=\int_{0}^{\varphi_{1}-\varphi_{10}} A(\varphi-e \xi) X(\varphi-e \xi) d \xi+\Psi\left(\hat{\varphi}-\hat{e}\left(\varphi_{1}-\varphi_{10}\right)\right)
$$

where $\Psi(\hat{\varphi})$ is a given $n \times n$ matrix, $X(\varphi)$ is the unknown $n \times n$ matrix, $\hat{\varphi}=\left(\varphi_{2}, \ldots, \varphi_{m}\right)$, and $\hat{e}=(1, \ldots, 1)$ is an $(m-1)$-vector. By $X\left(\varphi ; \varphi_{10}\right)$ we denote the solution of Eq. $(0.2)$ with $\Psi(\hat{\varphi})=E$ (the identity matrix) and $X_{0}(\varphi)=X(\varphi ; 0)$.

The following assertion was proved in [3].

Lemma 0.1. Let $A$ be a continuous $\omega$-periodic matrix function on $R^{m}$, and let $\Psi$ be a continuous matrix function nonsingular for all $\hat{\varphi} \in R^{m-1}$; then Eq. (0.2) has a unique solution, which can be 
represented in the form $X(\varphi)=X\left(\varphi ; \varphi_{10}\right) \Psi\left(\hat{\varphi}-\hat{e}\left(\varphi_{1}-\varphi_{10}\right)\right)$. The matrices $X(\varphi), X\left(\varphi ; \varphi_{10}\right)$, and $X_{0}(\varphi)$ have the following properties.

(a) $X(\varphi), X\left(\varphi ; \varphi_{10}\right)$, and $X_{0}(\varphi)$ are continuous matrix functions nonsingular for all $\varphi \in R^{m}$ and $\varphi_{10} \in R$.

(b) $X(\varphi ; s)=X(\varphi ; \tau) X\left(\varphi-e\left(\varphi_{1}-\tau\right) ; s\right), \varphi \in R^{m}, s, \tau \in R$.

(c) $X\left(\varphi ; \varphi_{10}\right)=X(\varphi) X^{-1}\left(\varphi-e\left(\varphi_{1}-\varphi_{10}\right)\right)=X_{0}(\varphi) X_{0}^{-1}\left(\varphi-e\left(\varphi_{1}-\varphi_{10}\right)\right)$, and $X\left(\varphi_{10}, \hat{\varphi} ; \varphi_{10}\right)=E$.

(d) $X\left(\varphi_{1}+\omega_{1}, \hat{\varphi} ; \varphi_{10}\right)=X\left(\varphi ; \varphi_{10}\right) X\left(\varphi_{10}+\omega_{1}, \hat{\varphi}-\hat{e}\left(\varphi_{1}-\varphi_{10}\right) ; \varphi_{10}\right)$; in particular,

$$
X_{0}\left(\varphi_{1}+\omega_{1}, \hat{\varphi}\right)=X_{0}(\varphi) X_{0}\left(\omega_{1}, \hat{\varphi}-\hat{e} \varphi_{1}\right) .
$$

[Here the vector $\varphi$ has the form $\left(\varphi_{1}, \hat{\varphi}\right)$.]

(e) The matrices $X\left(\varphi ; \varphi_{10}\right)$ and $X_{0}(\varphi)$ are $\hat{\omega}$-periodic in $\hat{\varphi}$ and the matrix $X$ is $\hat{\omega}$-periodic in $\hat{\varphi}$ if so is the matrix $\Psi$, where $\hat{\omega}=\left(\omega_{2}, \ldots, \omega_{m}\right)$.

(f) The relation

$$
\frac{d X\left(\varphi+e t ; \varphi_{10}\right)}{d t}=A(\varphi+e t) X\left(\varphi+e t ; \varphi_{10}\right)
$$

holds for arbitrary $\varphi \in R^{m}, \varphi_{10} \in R$, and $t \in R$; here $X_{0}($ et $)$ is the normalized principal solution matrix of system (0.1).

\section{INHOMOGENEOUS SYSTEM}

Consider the inhomogeneous system

$$
x^{\prime}=a(t) x+f(t),
$$

where $f$ is a continuous conditionally periodic vector function, $f(t)=F(e t), F \in P_{n}^{0}(\omega), P_{n}^{0}(\omega)$ is the Banach space of continuous $\omega$-periodic vector functions $F: R^{m} \rightarrow R^{n}$ with norm $\|F\|=$ $\sup _{\varphi \in R^{m}}|F(\varphi)|$, and $|\cdot|$ is a norm on the finite-dimensional space. The space of conditionally periodic functions $f(t)=F(e t), t \in R, F \in P_{n}^{0}(\omega)$, is denoted by $B_{n}^{0}(\omega)$.

Theorem 1.1. Let $f \in B_{n}^{0}(\omega)$. System (1.1) has a solution $x \in B_{n}^{0}(\omega)$ if and only if there exists a function $u_{0} \in P_{n}^{0}(\hat{\omega})$ such that the function

$$
u(\varphi)=X_{0}(\varphi) u_{0}\left(\hat{\varphi}-\hat{e} \varphi_{1}\right)+\int_{0}^{\varphi_{1}} X(\varphi ; \xi) F\left(\xi, \hat{\varphi}-\hat{e} \varphi_{1}+\hat{e} \xi\right) d \xi
$$

is an element of the space $P_{n}^{0}(\omega)$. The solution has the form $x(t)=u(e t)$.

Proof. Let $u \in P_{n}^{0}(\omega)$. We replace $\varphi$ in relation (1.2) by $\varphi+$ et and differentiate both sides of the resulting relation with respect to $t$,

$$
\begin{aligned}
\frac{d u(\varphi+e t)}{d t}= & A(\varphi+e t) X_{0}(\varphi+e t) u_{0}\left(\hat{\varphi}-\hat{e} \varphi_{1}\right)+X\left(\varphi+e t ; \varphi_{1}+t\right) F\left(\varphi_{1}+t, \hat{\varphi}+\hat{e} t\right) \\
& +\int_{0}^{\varphi_{1}+t} A(\varphi+e t) X(\varphi+e t ; \xi) F\left(\xi, \hat{\varphi}-\hat{e} \varphi_{1}+\hat{e} \xi\right) d \xi=A(\varphi+e t) u(\varphi+e t)+F(\varphi+e t) .
\end{aligned}
$$

Therefore, $u(e t)$ is a conditionally periodic solution of system (1.1).

Conversely, let $x$ be a conditionally periodic solution of system $(1.1), x(t)=u(e t)$; then for each $\tau \in R$, we have

$$
\frac{d u(e(t+\tau))}{d t}=A(e(t+\tau)) u(e(t+\tau))+F(e(t+\tau))
$$

DIFFERENTIAL EQUATIONS $\quad$ Vol. 49 No. $3 \quad 2013$ 
For each $\hat{\varphi} \in R^{m-1}$, there exists a sequence $\left\{\tau_{n}\right\}$ such that the sequences

$$
\left\{u\left(e\left(t+\tau_{n}\right)\right)\right\}, \quad\left\{A\left(e\left(t+\tau_{n}\right)\right)\right\}, \quad\left\{F\left(e\left(t+\tau_{n}\right)\right)\right\}
$$

converge uniformly on $R$ to $u(t, \hat{e} t+\hat{\varphi}), A(t, \hat{e} t+\hat{\varphi})$, and $F(t, \hat{e} t+\hat{\varphi})$, respectively, as $n \rightarrow+\infty$ $[1$, p. 340; 7, p. 126]; consequently,

$$
\frac{d u(t, \hat{e} t+\hat{\varphi})}{d t}=A(t, \hat{e} t+\hat{\varphi}) u(t, \hat{e} t+\hat{\varphi})+F(t, \hat{e} t+\hat{\varphi}) .
$$

The solution of this system can be represented in the form

$$
u(t, \hat{e} t+\hat{\varphi})=X_{0}(t, \hat{e} t+\hat{\varphi}) u(0, \hat{\varphi})+\int_{0}^{t} X(t, \hat{e} t+\hat{\varphi} ; \xi) F(\xi, \hat{e} \xi+\hat{\varphi}) d \xi .
$$

By setting $\psi_{1}=t, \hat{\psi}=\hat{\varphi}+\hat{e} t$, and $\psi=\left(\psi_{1}, \hat{\psi}\right)$, we obtain

$$
u(\psi)=X_{0}(\psi) u\left(0, \hat{\psi}-\hat{\psi}_{1}\right)+\int_{0}^{\psi_{1}} X(\psi ; \xi) F\left(\xi, \hat{e}\left(\xi-\psi_{1}\right)+\hat{\psi}\right) d \xi .
$$

We have thereby obtained a function $u \in P_{n}^{0}(\omega)$ of the form $(1.2)$ with $u_{0}(\hat{\varphi})=u(0, \hat{\varphi}) \in P_{n}^{0}(\hat{\omega})$. The proof of the theorem is complete.

Consider the monodromy operator $L$ of system (0.1), which is defined on the complex extension $\tilde{P}_{n}^{0}(\hat{\omega})$ of the space $P_{n}^{0}(\hat{\omega})$ by the formula [3] $L u=X_{0}\left(\omega_{1}, \hat{\varphi}\right) u\left(\hat{\varphi}-\hat{e} \omega_{1}\right)$. Obviously, this operator is linear, continuous, and continuously invertible. [One has $L^{-1} u=X_{0}^{-1}\left(\omega_{1}, \hat{\varphi}+\hat{e} \omega_{1}\right) u\left(\hat{\varphi}+\hat{e} \omega_{1}\right)$, the continuity of the matrix function $X_{0}^{-1}$ follows from the continuity and invertibility of the matrix function $X_{0}$.]

The following assertion was proved in [6].

Lemma 1.1. If a number $\lambda_{0}$ is a regular point of the operator $L$, then each number $\lambda$ such that $|\lambda|=\left|\lambda_{0}\right|$ is the regular point of $L$.

Lemma 1.2. The following relations hold for each $p \in Z$.

(a) $L^{p} u=X_{0}\left(p \omega_{1}, \hat{\varphi}\right) u\left(\hat{\varphi}-\hat{e} p \omega_{1}\right)$.

(b) $X_{0}\left(\varphi_{1}+p \omega_{1}, \hat{\varphi}\right)=X_{0}(\varphi) X_{0}\left(p \omega_{1}, \hat{\varphi}-\hat{e} \varphi_{1}\right), \varphi_{1} \in R, \hat{\varphi} \in R^{m-1}$.

Proof. Let $p \in N$; then, by induction, from item (d) of Lemma 0.1, we obtain the relations

$$
\begin{aligned}
L^{p} u & =X_{0}\left(\omega_{1}, \hat{\varphi}\right) X_{0}\left(\omega_{1}, \hat{\varphi}-\hat{e} \omega_{1}\right) \cdots X_{0}\left(\omega_{1}, \hat{\varphi}-\hat{e}(p-1) \omega_{1}\right) u\left(\hat{\varphi}-\hat{e} p \omega_{1}\right) \\
& =X_{0}\left(p \omega_{1}, \hat{\varphi}\right) u\left(\hat{\varphi}-\hat{e} p \omega_{1}\right)
\end{aligned}
$$

and

$$
\begin{aligned}
X_{0}\left(\varphi_{1}+p \omega_{1}, \hat{\varphi}\right) & =X_{0}(\varphi) X_{0}\left(\omega_{1}, \hat{\varphi}-\hat{e} \varphi_{1}\right) \cdots X_{0}\left(\omega_{1}, \hat{\varphi}-\hat{e}\left(\varphi_{1}+(p-1) \omega_{1}\right)\right) \\
& =X_{0}(\varphi) X_{0}\left(p \omega_{1}, \hat{\varphi}-\hat{e} \varphi_{1}\right) .
\end{aligned}
$$

It follows from the first relation that $L^{-p} u=X_{0}^{-1}\left(p \omega_{1}, \hat{\varphi}+\hat{e} p \omega_{1}\right) u\left(\hat{\varphi}+\hat{e} p \omega_{1}\right)$. In the second relation, we set $\varphi_{1}=-p \omega_{1}$; then $E=X_{0}\left(-p \omega_{1}+p \omega_{1}, \hat{\varphi}\right)=X_{0}\left(-p \omega_{1}, \hat{\varphi}\right) X_{0}\left(p \omega_{1}, \hat{\varphi}+\hat{e} p \omega_{1}\right)$ and $X_{0}^{-1}\left(p \omega_{1}, \hat{\varphi}+\hat{e} p \omega_{1}\right)=X_{0}\left(-p \omega_{1}, \hat{\varphi}\right)$. Consequently,

$$
L^{-p} u=X_{0}^{-1}\left(p \omega_{1}, \hat{\varphi}+\hat{e} p \omega_{1}\right) u\left(\hat{\varphi}+\hat{e} p \omega_{1}\right)=X_{0}\left(-p \omega_{1}, \hat{\varphi}\right) u\left(\hat{\varphi}+\hat{e} p \omega_{1}\right) .
$$

The proof of relation (a) is complete. Let us complete the proof of relation (b):

$$
X_{0}\left(\varphi_{1}-p \omega_{1}+p \omega_{1}, \hat{\varphi}\right)=X_{0}\left(\varphi_{1}-p \omega_{1}, \hat{\varphi}\right) X_{0}\left(p \omega_{1}, \hat{\varphi}-\hat{e}\left(\varphi_{1}-p \omega_{1}\right)\right) ;
$$

then

$$
X_{0}\left(\varphi_{1}-p \omega_{1}, \hat{\varphi}\right)=X_{0}(\varphi) X_{0}^{-1}\left(p \omega_{1}, \hat{\varphi}-\hat{e}\left(\varphi_{1}-p \omega_{1}\right)\right)=X_{0}(\varphi) X_{0}\left(-p \omega_{1}, \hat{\varphi}-\hat{e} \varphi_{1}\right) .
$$

The proof of the lemma is complete. 
Theorem 1.2. For each function $F \in P_{n}^{0}(\omega)$, there exists a unique function $u_{0} \in P_{n}^{0}(\hat{\omega})$ such that the function $u$ given by (1.2) is an element of the space $P_{n}^{0}(\omega)$ if and only if the spectrum of the operator $L$ does not meet the unit circle $|\lambda|=1$.

Proof. Necessity. Suppose that, for each function $F \in P_{n}^{0}(\omega)$, there exists a function $u_{0} \in P_{n}^{0}(\hat{\omega})$ described in the theorem; then

$$
0=u\left(\omega_{1}, \hat{\varphi}\right)-u_{0}(\hat{\varphi})=X_{0}\left(\omega_{1}, \hat{\varphi}\right) u_{0}\left(\hat{\varphi}-\hat{e} \omega_{1}\right)+\int_{0}^{\omega_{1}} X\left(\omega_{1}, \hat{\varphi} ; \xi\right) F\left(\xi, \hat{\varphi}-\hat{e} \omega_{1}+\hat{e} \xi\right) d \xi-u_{0}(\hat{\varphi}),
$$

whence we obtain the relation

$$
X_{0}\left(\omega_{1}, \hat{\varphi}\right) u_{0}\left(\hat{\varphi}-\hat{e} \omega_{1}\right)-u_{0}(\hat{\varphi})=-\int_{0}^{\omega_{1}} X\left(\omega_{1}, \hat{\varphi} ; \xi\right) F\left(\xi, \hat{\varphi}-\hat{e} \omega_{1}+\hat{e} \xi\right) d \xi .
$$

From item (c) of Lemma 0.1, we have $X\left(\omega_{1}, \hat{\varphi} ; \xi\right)=X_{0}\left(\omega_{1}, \hat{\varphi}\right) X_{0}^{-1}\left(\xi, \hat{\varphi}-\hat{e} \omega_{1}+\hat{e} \xi\right)$. For $F$, we take the function

$$
F(\varphi)=-\frac{6}{\omega_{1}^{3}} X_{0}(\varphi) X_{0}^{-1}\left(\omega_{1}, \hat{\varphi}-\hat{e} \varphi_{1}+\hat{e} \omega_{1}\right) h\left(\hat{\varphi}-\hat{e} \varphi_{1}+\hat{e} \omega_{1}\right) \varphi_{1}\left(\omega_{1}-\varphi_{1}\right),
$$

$\varphi_{1} \in\left[0, \omega_{1}\right]$, and we continue it as a periodic function of $\varphi_{1}$ outside the interval $\left[0, \omega_{1}\right]$, where $h$ is an arbitrary function from $P_{n}^{0}(\hat{\omega})$; then

$$
X_{0}\left(\omega_{1}, \hat{\varphi}\right) u_{0}\left(\hat{\varphi}-\hat{e} \omega_{1}\right)-u_{0}(\hat{\varphi})=h(\hat{\varphi}) .
$$

It follows from the existence and uniqueness of the solution of this equation for an arbitrary right-hand side that $\lambda=1$ is a regular point of the operator $L$. By Lemma 1.1, all points lying on the unit circle are regular.

Sufficiency. Suppose that the spectrum of $L$ does not meet the unit circle. The right-hand side of system (1.3) for each function $F \in P_{n}^{0}(\omega)$ is an element of the space $P_{n}^{0}(\hat{\omega})$; therefore, this system has a unique solution $u_{0} \in P_{n}^{0}(\hat{\omega})$. Consider the function $u$ given by $(1.2)$, where $u_{0}$ is a solution of system (1.3). This function is periodic in $\hat{\varphi}$ and continuous in $\varphi \in R^{m}$ by virtue of the periodicity and continuity of the right-hand side of (1.2). Let us show that it is periodic in $\varphi_{1}$. For an arbitrary fixed $\hat{\varphi}$, the functions $u(t, \hat{e} t+\hat{\varphi})$ and $u\left(t+\omega_{1}, \hat{e} t+\hat{\varphi}\right)$ satisfy system $\left(1.1_{\hat{\varphi}}\right)$; moreover, since $u\left(\omega_{1}, \hat{\varphi}\right)=u(0, \hat{\varphi})$, we have $u(t, \hat{e} t+\hat{\varphi})=u\left(t+\omega_{1}, \hat{e} t+\hat{\varphi}\right)$ by virtue of the uniqueness of the solution for all $t \in R$. Hence, it follows that $u\left(\varphi_{1}+\omega_{1}, \hat{\varphi}\right)=u(\varphi), \varphi \in R^{m}$; therefore, $u \in P_{n}^{0}(\omega)$. The proof of the theorem is complete.

\section{HOMOGENEOUS SYSTEM}

In forthcoming considerations, we need the following definition.

Definition 2.1 [4, p. 31; 5, p. 233]. System (0.1) is said to be exponentially dichotomous if the space $H=H(t)$ of its solutions can be represented as the direct sum $H_{1} \dot{+} H_{2}$ of subspaces $H_{1}=H_{1}(t)$ and $H_{2}=H_{2}(t)$ and there exist positive constants $M_{1}, M_{2}, \gamma_{1}$, and $\gamma_{2}$ such that

$$
|x(t)| \leq M_{1} e^{-\gamma_{1}(t-\tau)}|x(\tau)|, \quad-\infty<\tau \leq t<+\infty,
$$

for $x \in H_{1}$ and

$$
|x(t)| \geq M_{2} e^{\gamma_{2}(t-\tau)}|x(\tau)|, \quad-\infty<\tau \leq t<+\infty,
$$

for $x \in H_{2}$.

Theorem 2.1. System (0.1) is exponentially dichotomous if and only if the spectrum of the operator $L$ does not meet the unit circle.

DIFFERENTIAL EQUATIONS $\quad$ Vol. 49 No. 32013 
Proof. Necessity. If system (0.1) is exponentially dichotomous then, for each continuous conditionally periodic function $f$, system (1.1) has a unique continuous almost periodic solution [5, pp. 274-275]. Since the module of this solution lies in the minimum module containing the union of the spectra of the functions $f$ and $a$ [5, p. 275], it follows that such a solution is conditionally periodic with frequencies $\beta_{1}, \ldots, \beta_{m}$. Then the spectrum of $L$ does not meet the unit circle by Theorems 1.1 and 1.2 .

Sufficiency. If the spectrum $\sigma$ of $L$ does not meet the unit circle, then $\sigma=\sigma_{1} \cup \sigma_{2}$, where $\sigma_{1}$ is the part of the spectrum lying inside the unit circle and $\sigma_{2}$ is the part lying outside it. The space $P_{n}^{0}(\hat{\omega})$ can be represented as the direct sum of invariant subspaces $P_{n 1}^{0}(\hat{\omega})$ and $P_{n 2}^{0}(\hat{\omega})$ of the operator $L, P_{n k}^{0}(\hat{\omega})=\Pi_{k} P_{n}^{0}(\hat{\omega})$, where the $\Pi_{k}$ are the spectral projections of the operator $L$ corresponding to $\sigma_{k}, k=1,2$.

Let $L_{k}$ be the restriction of the operator $L$ to the subspace $P_{n k}^{0}(\hat{\omega})$; then $[5$, p. 290] there exist numbers $K>0$ and $q \in(0,1)$ such that

$$
\left\|L_{1}^{s}\right\| \leq K q^{s}, \quad\left\|L_{2}^{-s}\right\| \leq K q^{s}, \quad s \in N .
$$

Let $x_{0} \in P_{n 1}^{0}(\hat{\omega}), t=\xi+\tau, \xi=\xi_{1}+r \omega_{1}, \tau=\tau_{1}+p \omega_{1}, \xi_{1}, \tau_{1} \in\left[0, \omega_{1}\right], r, p \in Z$, and $r \geq 0$. The vector function $x\left(t, \hat{\varphi}, x_{0}\right)=X_{0}(t, \hat{e} t+\hat{\varphi}) x_{0}(\hat{\varphi})$ with $\hat{\varphi}=0$ is a solution of system (0.1) [and, for an arbitrary $\hat{\varphi}$, is a solution of system $\left(1.1_{\hat{\varphi}}\right)$, where $\left.f=0\right]$.

To prove inequality (2.1), we use Lemma 1.2, the inequality $\left[4\right.$, p. 32] $\left|x\left(t, 0, x_{0}\right)\right| \leq c_{1}\left|x\left(\zeta, 0, x_{0}\right)\right|$, which holds for some $c_{1}$ and for all $t$ and $\zeta$ such that $|t-\zeta| \leq 2 \omega_{1}$, and the inequality

$$
\left\|X_{0}\left(\xi_{1}+\tau_{1}, \hat{\varphi}\right)\right\| \leq c_{2},
$$

which holds for some $c_{2}$ for any $\hat{\varphi}$ and $\left|\xi_{1}+\tau_{1}\right| \leq 2 \omega_{1}$; then we obtain

$$
\begin{aligned}
\left|x\left(t, 0, x_{0}\right)\right| & =\left|X_{0}(e t) x_{0}(0)\right| \leq\left\|X_{0}(t, \hat{e} t+\hat{\varphi}) x_{0}(\hat{\varphi})\right\| \\
& =\left\|X_{0}\left(\xi_{1}+\tau_{1}, \hat{e} t+\hat{\varphi}\right) X_{0}\left((r+p) \omega_{1}, \hat{e}(r+p) \omega_{1}+\hat{\varphi}\right) x_{0}(\hat{\varphi})\right\| \\
& \leq c_{2}\left\|X_{0}\left((r+p) \omega_{1}, \hat{e}(r+p) \omega_{1}+\hat{\varphi}\right) x_{0}(\hat{\varphi})\right\|=c_{2}\left\|X_{0}\left((r+p) \omega_{1}, \hat{\varphi}\right) x_{0}\left(\hat{\varphi}-\hat{e}(r+p) \omega_{1}\right)\right\| \\
& =c_{2}\left\|L_{1}^{r+p} x_{0}\right\| \leq c_{2} K q^{r}\left\|L_{1}^{p} x_{0}\right\|=c_{2} K e^{-\xi_{1} \omega_{1}^{-1} \ln q} e^{\xi \omega_{1}^{-1} \ln q}\left\|X_{0}\left(p \omega_{1}, \hat{e} p \omega_{1}+\hat{\varphi}\right) x_{0}(\hat{\varphi})\right\| \\
& \leq\left(c_{2} K e^{-\xi_{1} \omega_{1}^{-1} \ln q} \leq c_{3}, \gamma_{1}=-\omega_{1}^{-1} \ln q>0\right) \leq c_{3} e^{-\gamma_{1} \xi}\left\|x\left(p \omega_{1}, \hat{\varphi}, x_{0}\right)\right\| .
\end{aligned}
$$

In a similar way, one can prove the inequality

$$
\left|x\left(\tau, 0, x_{0}\right)\right| \leq c_{4} e^{-\gamma_{2} \xi}\left\|x\left((p+r) \omega_{1}, \hat{\varphi}, x_{0}\right)\right\|,
$$

where $\gamma_{2}=-\omega_{1}^{-1} \ln q>0$ and $x_{0} \in P_{n 2}^{0}(\hat{\omega})$.

It follows from inequalities (2.4) and (2.5) that the expansion $x_{0}=x_{01}+x_{02}, x_{0 k}=\Pi_{k} x_{0}$, of an arbitrary element $x_{0} \in P_{n}^{0}(\hat{\omega})$ implies the expansion $x_{0}(0)=x_{01}(0)+x_{02}(0)$ of an arbitrary element $x_{0}(0) \in R^{n}$. This expansion is unique. Indeed, if we assume that $\tilde{x}_{0} \neq x_{0}, \tilde{x}_{0}(0)=x_{0}(0)$ and $\tilde{x}_{0}=\tilde{x}_{01}+\tilde{x}_{02}, \tilde{x}_{0 k}=\Pi_{k} \tilde{x}_{0}$, then $x_{01}(0)-\tilde{x}_{01}(0)=\tilde{x}_{02}(0)-x_{02}(0)$, and for $x_{01}(0)-\tilde{x}_{01}(0) \neq 0$ the solution of system (0.1) equal to $x_{01}-\tilde{x}_{01}$ for $t=0$ is both infinitely large and infinitely small as $t \rightarrow+\infty$.

Therefore, the space $R^{n}$ can be represented as the direct sum of subspaces $H_{1}(0)$ and $H_{2}(0)$; moreover, the solutions of system (0.1) issuing for $t=0$ from the subspace $H_{1}(0)$ tend to zero, and those issuing from $H_{2}(0)$ tend to infinity as $t \rightarrow+\infty$. Likewise, if, instead of $\hat{\varphi}=0$, we fix an arbitrary $\hat{\varphi}_{0} \in R^{m-1}$, then the expansion

$$
x_{0}\left(\hat{\varphi}_{0}\right)=x_{01}\left(\hat{\varphi}_{0}\right)+x_{02}\left(\hat{\varphi}_{0}\right), \quad x_{0 k}\left(\hat{\varphi}_{0}\right)=\left(\Pi_{k} x_{0}\right)\left(\hat{\varphi}_{0}\right)
$$

is independent of the choice of the function $x_{0}$ and depends only on the value taken by this function at the point $\hat{\varphi}_{0}$. In particular, hence, it follows that if $x_{0} \in P_{n 1}^{0}(\hat{\omega})$ and $y \neq x_{0}$, but $y\left(\hat{\varphi}_{0}\right)=x_{0}\left(\hat{\varphi}_{0}\right)$, then $\left(\Pi_{1} y\right)\left(\hat{\varphi}_{0}\right)=y\left(\hat{\varphi}_{0}\right)$. 
Let us return to inequality $(2.4)$, where $x_{0} \in P_{n 1}^{0}(\hat{\omega})$ and $x\left(p \omega_{1}, \hat{\varphi}, x_{0}\right)=\left(L_{1}^{p} x_{0}\right)\left(\hat{e} p \omega_{1}+\hat{\varphi}\right)$. Set $\tilde{x}_{0}=L_{1}^{-p} \Pi_{1} x_{0}\left(p \omega_{1}, 0, x_{0}\right) \in P_{n 1}^{0}(\hat{\omega})$. (The argument of the operator $\Pi_{1}$ is a constant function of $\hat{\varphi}$.) Then

$$
x\left(p \omega_{1}, \hat{\varphi}, \tilde{x}_{0}\right)=\left(L_{1}^{p} \tilde{x}_{0}\right)\left(\hat{\varphi}+p \omega_{1} \hat{e}\right)=\left(\Pi_{1} x\left(p \omega_{1}, 0, x_{0}\right)\right)\left(\hat{\varphi}+p \omega_{1} \hat{e}\right),
$$

and since

$$
\begin{aligned}
x\left(p \omega_{1}, 0, \tilde{x_{0}}\right) & =\left(\Pi_{1} x\left(p \omega_{1}, 0, x_{0}\right)\right)\left(p \omega_{1} \hat{e}\right) \\
& =\left(\Pi_{1}\left(\left(L_{1}^{p} x_{0}\right)\left(p \omega_{1} \hat{e}\right)\right)\right)\left(p \omega_{1} \hat{e}\right)=\left(L_{1}^{p} x_{0}\right)\left(p \omega_{1} \hat{e}\right)=x\left(p \omega_{1}, 0, x_{0}\right),
\end{aligned}
$$

it follows from the uniqueness of the solution of system $(0.1)$ that $\tilde{x}_{0}(0)=x_{0}(0)$. Let us replace $x_{0}$ in inequality $(2.4)$ by $\tilde{x}_{0}$; then

$$
\begin{aligned}
\left|x\left(t, 0, x_{0}\right)\right| & =\left|x\left(t, 0, \tilde{x}_{0}\right)\right| \leq c_{3} e^{-\gamma_{1} \xi}\left\|x\left(p \omega_{1}, \hat{\varphi}, \tilde{x}_{0}\right)\right\|=c_{3} e^{-\gamma_{1} \xi}\left\|\Pi_{1} x\left(p \omega_{1}, 0, x_{0}\right)\right\| \\
& \leq c_{3} e^{-\gamma_{1} \xi}\left\|\Pi_{1}\right\|\left|x\left(p \omega_{1}, 0, x_{0}\right)\right| \leq c_{3} c_{1} e^{-\gamma_{1} \xi}\left\|\Pi_{1}\right\|\left|x\left(\tau, 0, x_{0}\right)\right| .
\end{aligned}
$$

The proof of inequality (2.1) is complete; inequality (2.2) can be proved in a similar way. The proof of the theorem is complete.

Note that the monodromy operator $L_{\varphi_{0}}$ of the system

$$
\frac{d x}{d t}=A\left(e t+\varphi_{0}\right) x
$$

given by the relation $L_{\varphi_{0}} u=X\left(\varphi_{10}+\omega_{1}, \hat{\varphi}+\hat{\varphi}_{0} ; \varphi_{10}\right) u\left(\hat{\varphi}-\hat{e} \omega_{1}\right)$ is similar to the operator $L$ for any $\varphi_{0} \in R^{m}$; consequently, their spectra coincide. To prove the desired assertion, consider the shift operator $S_{\varphi_{0}} u=u\left(\hat{\varphi}+\hat{\varphi}_{0}\right)$ and the operator of multiplication by the matrix $\mathcal{X} u=X_{0}\left(\varphi_{10}, \hat{\varphi}\right) u(\hat{\varphi})$, $u \in \tilde{P}_{n}^{0}(\hat{\omega})$. It follows from Lemma 0.1 that

$$
X\left(\varphi_{10}+\omega_{1}, \hat{\varphi}+\hat{\varphi}_{0} ; \varphi_{10}\right)=X_{0}\left(\varphi_{10}, \hat{\varphi}+\hat{\varphi}_{0}\right) X_{0}\left(\omega_{1}, \hat{\varphi}+\hat{\varphi}_{0}-\hat{e} \varphi_{10}\right) X_{0}^{-1}\left(\varphi_{10}, \hat{\varphi}+\hat{\varphi}_{0}-\hat{e} \omega_{1}\right)
$$

therefore, $L_{\varphi_{0}}=S_{\hat{\varphi}_{0}} \mathcal{X} S_{\hat{e} \varphi_{10}}^{-1} L S_{\hat{e} \varphi_{10}} \mathcal{X}^{-1} S_{\hat{\varphi}_{0}}^{-1}$. We have thereby proved the following assertion.

Theorem 2.2. System (2.6) is exponentially dichotomous for any $\varphi_{0} \in R^{m}$ if and only if the spectrum of the operator $L$ does not meet the unit circle.

\section{REFERENCES}

1. Bohl, P., Sobranie trudov (Collected Works), Riga: Zinatne, 1974.

2. Kharasakhal, V.Kh., Pochti-periodicheskie resheniya obyknovennykh differentsial'nykh uravnenii (Almost Periodic Solutions of Ordinary Differential Equations), Alma-Ata: Nauka, 1970.

3. Kozlov, Yu.D., On the Reducibility of Linear Differential Equations with Conditionally Periodic Coefficients, Deposited in VINITI, Yekaterinburg, 1995, no. 1278-V95.

4. Krasnosel'skii, M.A., Burd, V.Sh., and Kolesov, Yu.S., Nelineinye pochti-periodicheskie kolebaniya (Nonlinear Almost Periodic Oscillations), Moscow: Nauka, 1970.

5. Daletskii, Yu.L. and Krein, M.G., Ustoichivost' reshenii differentsial'nykh uravnenii v banakhovom prostranstve (Stability of Solutions of Differential Equations in Banach Space), Moscow: Nauka, 1970.

6. Antonevich, A.B., Lineinye funktsional'nye uravneniya: Operatornyi podkhod (Linear Functional Equations. The Operator Approach), Minsk: Universitetskoe, 1988.

7. Levitan, B.M., Pochti-periodicheskie funktsii (Almost Periodic Functions), Moscow: Gosudarstv. Izdat. Tekhn.-Teor. Lit., 1953. 